\title{
Measurements of the top quark properties at production with CMS
}

\section{Mykola Savitskyi*i}

Deutsches Elektronen-Synchrotron (DE)

E-mail: mykola.savitskyi@cern.ch

\begin{abstract}
Several measurements of top quark properties are presented using data collected by the CMS experiment at the centre-of-mass-energy of $8 \mathrm{TeV}$. The properties are mostly probed in production of the top quarks. The charge asymmetry is measured inclusively and differentially probing anomalous couplings to the gluons at production mode. These measurements are extended searching for asymmetries in CP-odd operators in production and decay. The measurement of the spin density matrix in different final states (lepton+jets and dileptons) is discussed as well as the measurement of the top quark polarization in $t \bar{t}$ events. The results are compared to the state-of-the-art predictions, when available, and re-interpreted as searches for new physics inducing deviations from the standard model predictions.
\end{abstract}

The European Physical Society Conference on High Energy Physics

5-12 July, 2017

Venice, Italy

${ }^{*}$ Speaker.

${ }^{\dagger}$ On behalf of the CMS collaboration. 


\section{Introduction}

The top quark is the heaviest elementary particle known to-date. Because of its large mass, the top quark plays a crucial role in electroweak loop corrections, providing indirect constraints on the mass of the Higgs boson. Also, it is the only quark that decays before forming hadrons. This offers a unique opportunity to measure directly the properties of a "bare" quark. Precise measurements of top quark properties provide important input to standard model (SM) QCD predictions and can reveal signs of new physics phenomena as deviations from the SM expectations.

Top quarks are produced at the LHC mostly in pairs $(t \bar{t})$ via the strong interaction. The top quark decays almost exclusively into a $\mathrm{W}$ boson and a $\mathrm{b}$ quark. The subsequent decay of the $\mathrm{W}$ bosons define the $t \bar{t}$ final states: two leptons, two neutrinos and two $\mathrm{b}$ jets (dilepton channel), one lepton, one neutrino and four jets, out of which two arise from a $b$ quark (lepton+jets channel) or with six jets, out of which two stem from a $b$ quark (all hadronic channel).

In this document, I present a review of the most recent results on top quark properties in $t \bar{t}$ production, such as top quark spin correlation and polarization, $t \bar{t}$ charge asymmetry, and search for CP-violation. The measurements are performed by the CMS Collaboration [1] in the dilepton and $1+$ jets final states, using data from the LHC at a centre-of-mass energy $\sqrt{s}=8 \mathrm{TeV}$.

\section{Spin Correlations and Polarization}

In the SM, the top quarks from pair production are almost completely unpolarized, but they have the correlated spins. The top quark lifetime $\left(\sim 10^{-25} \mathrm{~s}\right)$ is much shorter than the spin decorrelation time scale $\left(\sim 10^{-21} \mathrm{~s}\right)$. Thus, the angular distributions of the top quark decay products provide access to spin of corresponding top quark.

The SM assumptions behind the spin correlation scenario are studied in terms of the spin correlation strength $A=\frac{\left(N_{\uparrow \uparrow}+N_{\downarrow \downarrow}\right)-\left(N_{\uparrow \downarrow}+N_{\downarrow \uparrow}\right)}{\left(N_{\uparrow \uparrow}+N_{\downarrow \downarrow}\right)+\left(N_{\uparrow \downarrow}+N_{\downarrow \uparrow}\right)}$ and the fraction coefficient $f_{S M}=\frac{N_{S M}^{t i}}{N_{S M}^{t \bar{t}}+N_{\text {uncorrelated }}^{t \bar{t}}}$. The spin correlation strength $A$ is probed in terms of the asymmetry between the number of $t \bar{t}$ pairs with aligned and antialigned spins. The fraction coefficient shows the fraction of $t \bar{t}$ events with the spin correlation according to SM prediction.

\section{Results in Dilepton Final States}

In the measurement [2], the coefficients of spin density matrix and $f_{S M}$ are probed using the asymmetries in angular observables of two leptons.

The inclusive asymmetries are extracted from the normalized differential cross sections which are measured in bins of $\Delta \phi_{\ell^{+} \ell^{-}}$(the difference in azimuthal angles in the laboratory frame), $\cos \theta_{\ell^{ \pm}}^{*}$ and $\cos \theta_{\ell^{+}}^{*} \cdot \cos \theta_{\ell^{-}}^{*}$ (the variables that are dependent from the lepton helicity angles $\theta^{*}$ ), $\cos \phi$ (the angle between leptons that are measured in the rest frames of parental top quarks). For example, the measured inclusive asymmetry $A_{\Delta \phi_{\ell^{+} \ell^{-}}}=0.094 \pm 0.005$ (stat.) \pm 0.012 (syst.) yields $f_{S M}=1.14_{-0.18}^{+0.16}($ tot. $)$. In addition, the asymmetries are studied as a function of $m_{t \bar{t}},\left|y_{t \bar{t}}\right|, p_{T}^{t \bar{t}}$. The examples of aforementioned cross section measurements and differential asymmetries are shown in Figure 1. All measurements are in agreement with the SM expectations. 

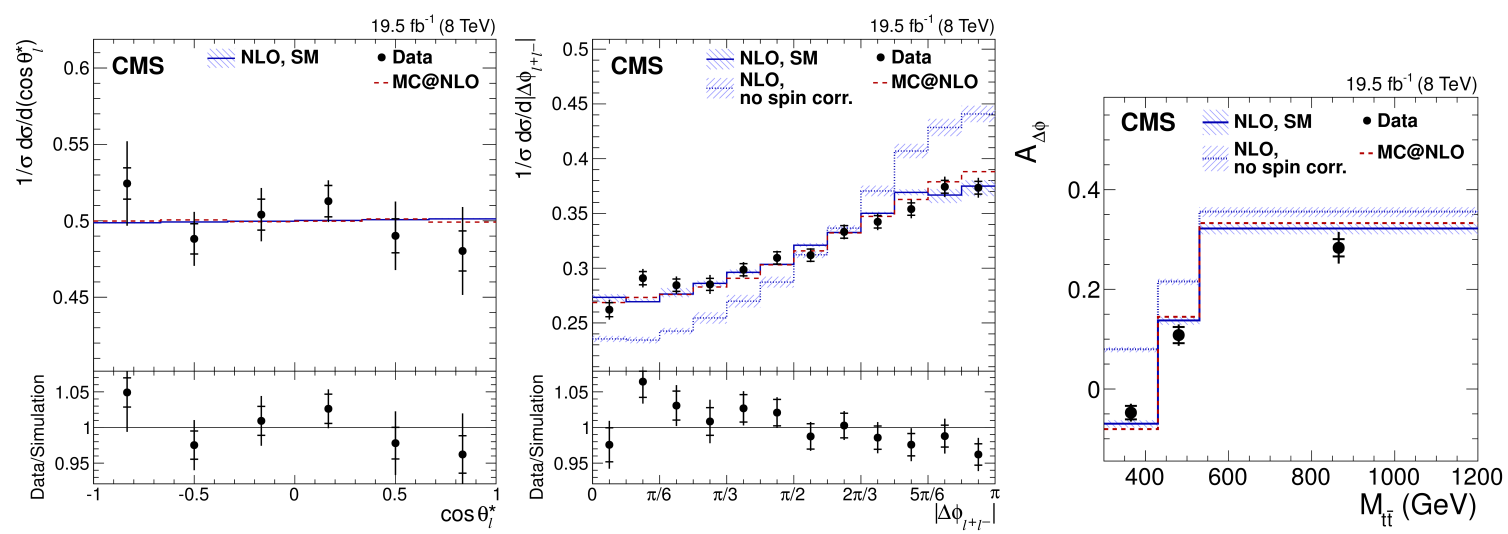

Figure 1: Normalized differential cross section in bins of $\cos \theta_{\ell^{ \pm}}^{*}$ (left) and of $\Delta \phi_{\ell^{+} \ell^{-}}$(middle). Differential asymmetry $A_{\Delta \phi_{\ell^{+} \ell^{-}}}$as a function of $m_{t \bar{t}}$ (right). The results are taken from [2].

\section{Results in Muon+Jets Final States}

Measurement [3] checks the consistency of spin correlation strength in $t \bar{t}$ events with the SM scenario. For doing so, the two hypotheses behind the $t \bar{t}$ production in data are tested, which assume either the spin correlations as they are predicted by the SM or their absence. This is done with the event probability estimation technique involving the matrix element method. The separation power between the two hypotheses is given by the sample likelihood ratios $\lambda_{\text {sample }}$ for the data and corresponding simulated samples to one of the hypotheses (Figure 2, left). It is found that the SM correlation agrees with the data within 2.2 standard deviation, while the assumption of no spin correlations is localized in the interval of 2.9 standard deviation.

Additionally, the extraction of $f_{S M}$ is performed using the distribution of event likelihood ratio $\lambda_{\text {event }}$ (Figure 2, right). The SM constribution is $f_{S M}=0.72 \pm 0.08(\text { stat } .)_{-0.13}^{+0.15}($ syst .). The spin correlation strength is estimated from simulation and found to be $A_{\text {hel }}^{\text {data }}=f_{S M} \cdot A_{\text {hel }}^{S M, M C}=$ $0.23 \pm 0.03(\text { stat. })_{-0.04}^{+0.05}($ syst. $)$.
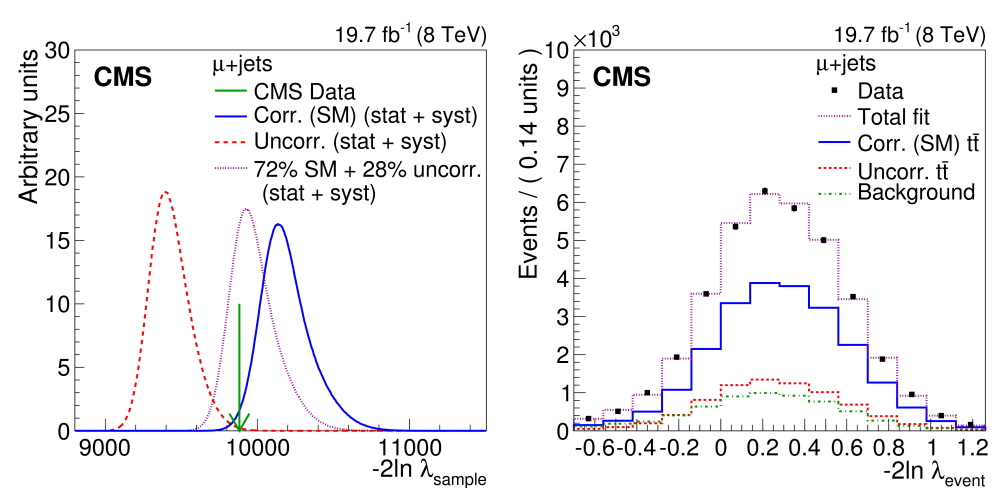

Figure 2: The related distributions to the sample likelihood ratio (left) and event likelihood ratio (right) for the data and tested hypotheses behind $t \bar{t}$ production in [3]. 


\section{Charge Asymmetry}

The SM predicts a non-zero (but small) charge asymmetry in $t \bar{t}$ production that occurs through the $q \bar{q}$ interaction at beyond-LO accuracy. At the LHC, the different centrality in rapidity $y$ is observed for top quarks with respect to anti-quarks. Therefore, the charge asymmetry can be measured as $A_{C}=\frac{N\left(\Delta\left|y_{t}\right|>0\right)-N\left(\Delta\left|y_{t}\right|<0\right)}{N\left(\Delta\left|y_{t}\right|>0\right)+N\left(\Delta\left|y_{t}\right|<0\right)}$, where $\Delta\left|y_{t}\right|=\left|y_{t}\right|-\left|y_{\bar{t}}\right|$.

\section{Results in Dilepton Final States}

Inclusive and differential measurements of $A_{C}$ are performed in [4]. These results are complemented with the asymmetry $A_{C}^{l e p}$ that is based on the leptonic observables and constructed in a similar way as $A_{C}$, but using instead the variable $\Delta\left|\eta_{\ell}\right|=\left|\eta_{\ell^{+}}\right|-\left|\eta_{\ell^{-}}\right|$. The $A_{C}^{\text {lep }}$ asymmetry has the advantage that it has better resolution and does not require a kinematic reconstruction of the top-quark pair; however, the effects of the asymmetry are more diluted through the top quark decay products. Moreover, it carries the additional information regarding the top quark polarization.

The inclusive asymmetries are computed from the respective normalized differential cross sections (see, as an example, Figure 3, left). The measured asymmetries $A_{C}=0.011 \pm 0.011$ (stat.) \pm 0.007 (syst.) and $A_{C}^{\text {lep }}=0.003 \pm 0.006$ (stat.) \pm 0.003 (syst.) are consistent with the expectations from the SM.

The differential asymmetries are extracted from the unfolded spectra in bins of $m_{t \bar{t}},\left|y_{t \bar{t}}\right|, p_{T}^{t \bar{t}}$. Figure 3 depicts the differential $A_{C}$ and $A_{C}^{l e p}$ measurements as the functions of $m_{t \bar{t}}$.
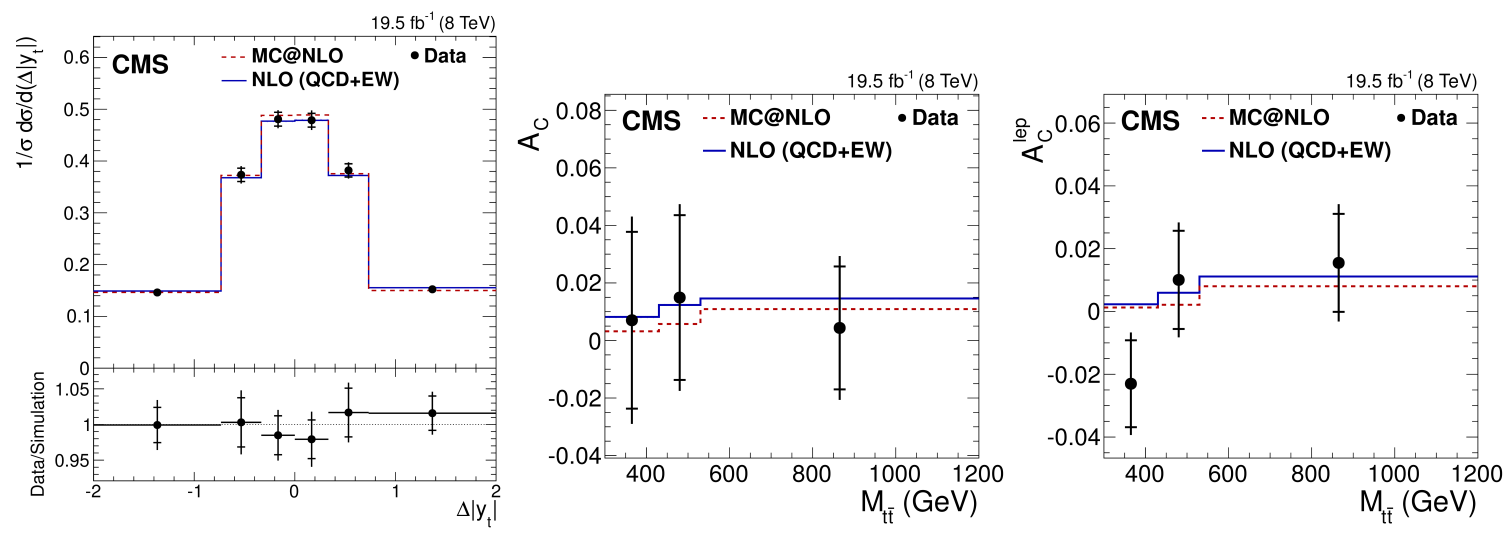

Figure 3: The normalized differential cross sections in bins of $\Delta\left|y_{t}\right|$ (left). The differential asymmetries $A_{C}$ (middle) and $A_{C}^{l e p}$ (right) as the functions of $m_{t \bar{t}}$. The measurements are performed in the dilepton channel [4].

\section{Results in Lepton+Jets Final States}

The inclusive and differential charge asymmetries $A_{C}$ are also measured in the $1+$ jets channel using a similar strategy as described above for the dilepton channel [5]. However, the results are presented in the full and fiducial phase spaces (PS). The differential measurement of $A_{C}$ in bins of $\left|y_{t i t}\right|$ after the extrapolation to full PS is displayed in Figure 4 (left).

The measurement [6] also performs the extraction of inclusive $A_{C}$, but performing a template fit to the reconstructed values of transformed rapidity observable $\Upsilon_{t \bar{t}}=\tanh \left(\Delta\left|y_{t}\right|\right)$. The fitting 
method involves the (anti)symmetric $x^{(-)+}$components of the predicted probability density for $\Upsilon_{t \bar{t}}$ in the base model (SM), which are related according to $x_{\alpha}^{\text {data }}=x^{+, r e c}+\alpha \cdot x^{-, \text {rec }}$. The result of this extraction is shown in Figure 4.
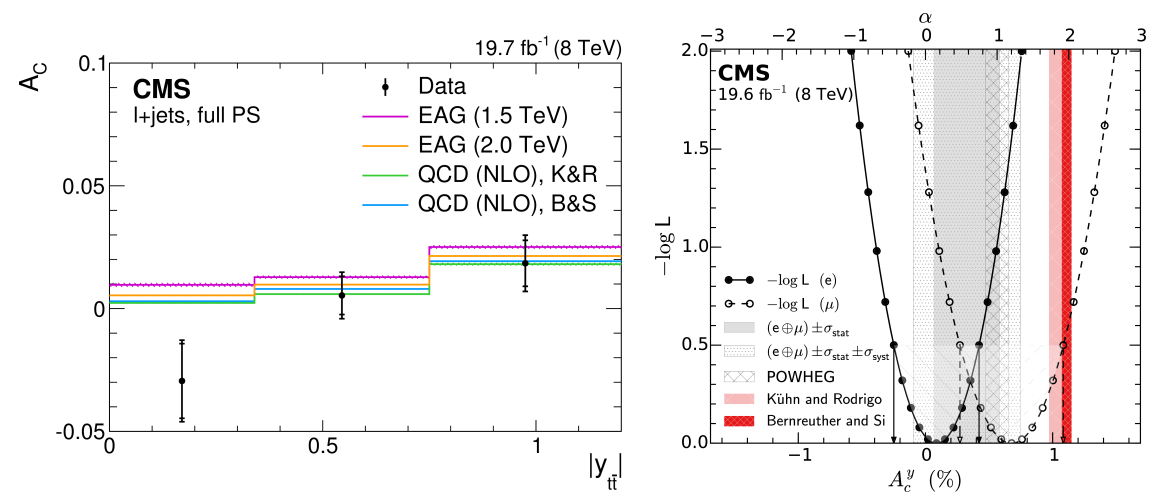

Figure 4: Differential measurement of charge asymmetry $A_{C}$ in bins of $\left|y_{t t}\right|$ in the 1+jets channel (left) [5]. Inclusive $A_{C}$ extraction in the l+jets channel with the template method (right) [6].

\section{CP Violation in Top-Quark Pair Production and Decay}

$\mathrm{CP}$ violation (CPV) is predicted to be very small in the SM, but it may be enhanced by potential new physics phenomena. In $t \bar{t}$ events, one can search for the effects induced by CP-violating anomalous top quark couplings.

The first measurement of CP-violating asymmetries in $t \bar{t}$ production and decay is performed in [7]. For this purpose, the four T-odd observables $O_{i}$ are constructed using the spin or momentum vectors of the top quark decay products in lepton+jets final state, e.g. $O_{2}=\left(\vec{p}_{b}+\vec{p}_{\bar{b}}\right) \cdot\left(\vec{p}_{\ell} \times \vec{p}_{j_{1}}\right)$ (see Figure 5). The non-zero asymmetry in $O_{i}$ serves as an evidence of CPV. These asymmetries are computed as $A_{C P}\left(O_{i}\right)=\frac{N\left(O_{i}>0\right)-N\left(O_{i}<0\right)}{N\left(O_{i}>0\right)+N\left(O_{i}<0\right)}$. Depending on the theory model, the up-to $8 \%$ variations due to CPV effects are expected in the values of asymmetries. All the measured $A_{C P}\left(O_{i}\right)$ are consistent with the SM (see Figure 5).
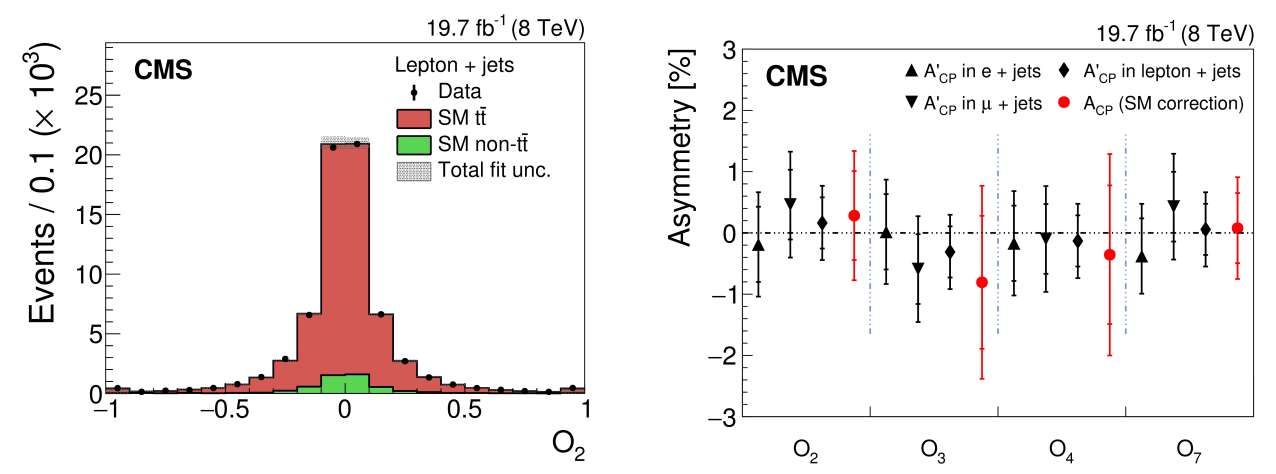

Figure 5: The example of T-odd observable $O_{2}$ (left). This and the other $O_{i}$ observables are used for the extraction of CP-violating asymmetries (right) in [7]. 


\section{Summary}

A review of the most recent measurements of top quark properties in $t \bar{t}$ production performed by the CMS Collaboration is presented. All results, which are obtained using the full set of data collected at $8 \mathrm{TeV}$, are found to be in good agreement with the SM expectations, and no hints of new physics phenomena is found. A next round of new exciting results will come with the examination of latest $13 \mathrm{TeV}$ data from LHC Run2.

\section{References}

[1] The CMS Collaboration, The CMS Experiment at the CERN LHC, JINST 3, (2008) S08004

[2] The CMS Collaboration, Measurements of $t \bar{t}$ spin correlations and top quark polarization using dilepton final states in pp collisions at $\sqrt{s}=8 \mathrm{TeV}$, Phys. Rev. D 93, 052007 (2016) [hep-ex/1601.01107v2]

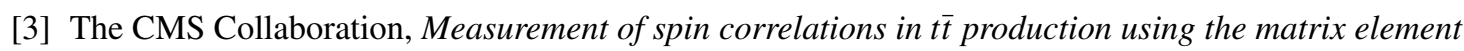
method in the muon+jets final state in pp collisions at $\sqrt{s}=8 \mathrm{TeV}$, Phys. Lett. B 758, (2016) 321 [hep-ex/1511.06170v2]

[4] The CMS Collaboration, Measurements of $t \bar{t}$ charge asymmetry using dilepton final states in $p p$ collisions at $\sqrt{s}=8 \mathrm{TeV}$, Phys. Lett. B 760, (2016) 365 [hep-ex/1603.06221v2]

[5] The CMS Collaboration, Inclusive and differential measurements of the $t \bar{t}$ charge asymmetry in pp collisions at $\sqrt{s}=8 \mathrm{TeV}$, Phys. Lett. B 757, (2016) 154 [hep-ex/1507.03119v2]

[6] The CMS Collaboration, Measurement of the charge asymmetry in top quark pair production in pp collisions at $\sqrt{s}=8$ TeVusing a template method, Phys. Rev. D 93, 034014 (2016) [hep-ex/1508.03862v1]

[7] The CMS Collaboration, Search for CP violation in $t \bar{t}$ production and decay in proton-proton collisions at $\sqrt{s}=8 \mathrm{TeV}, \mathrm{JHEP} \mathbf{0 3}$, (2017) 101 [hep-ex/1611.08931v2] 